\title{
Sclerosing Stromal Tumour of Ovary- A Case Report
}

\author{
FAHMIDA SHARMIN JOTY ${ }^{1}$,FARHANA DEWAN ${ }^{2}$, FAHMIDA KHAN LIMA ${ }^{3}$, \\ BIPUL BISWAS ${ }^{4}$, EVA RANI NONDI ${ }^{5}$
}

\begin{abstract}
:
Sclerosing stromal tumor (SST) is an extremely rare and distinctive sex cord stromal tumor, which occurs predominantly in the second and third decades of life. Approximately 100 cases reported since first described in 1973. It typically present with pelvic/abdominal pain and tenderness, mass, and/or abnormal menses, and with hormonal activity reported predominantly in postmenarchal females. Only 5 cases of these tumors have been reported in premenarchal girls. We report a case of a 18-year-old girl who developed a sclerosing stromal tumor of ovary and presented with excessive menstruation, pelvic pain and increased frequency of menstruation. Her hormonal status was normal. She was suspected to have a malignant tumor on computed tomography and underwent unilateral salpingo-oopherectomy. It is therefore, necessary to keep in mind the possibility of sclerosing stromal tumor in a young woman.
\end{abstract}

Key Words: Ovarian tumor, Sclerosing stromal tumor, sex cord stromal tumor, hyperestrogenism

\section{Introduction}

Sclerosing stromal tumor (SST) is an extremely rare benign subtype of ovarian stromal neoplasm of the sex cord stromal category is included in the fibromathecoma group of ovarian tumors that has distinctive clinical and pathological features, which differentiate it from other stromal tumors. The tumor occurs predominantly in the 2 nd and $3 r d$ decades of life ${ }^{1,2}$. It is usually unilateral and well circumscribed; its recurrence has not been reported ${ }^{3}$. Histologically it is characterized by a network of thin walled vessels, sclerosis, heterogeneity of the cellular areas and illdefined cellular pseudolobules separated by a densely hyalinised or markedly edematous stroma ${ }^{1}$.

\section{Case report}

A18-year-old unmarried girl presented in the in patient department of obstetrics and gynaecology with excessive cyclical bleeding \& pelvic pain for 6 months. Her menstrual period was 15-20 days for last 6 months. On clinical examination, she was moderately anaemic. A mass felt about $5 \times 5 \mathrm{~cm}$ in right iliac region, hard in consistency, smooth surfaced, ill defined margin, fixed. Ultrasonography showed a mixed echogenic mass seen in right adnexal region measuring about $5 \times 5.7 \mathrm{~cm}$ suggestive of right adnexal mass.

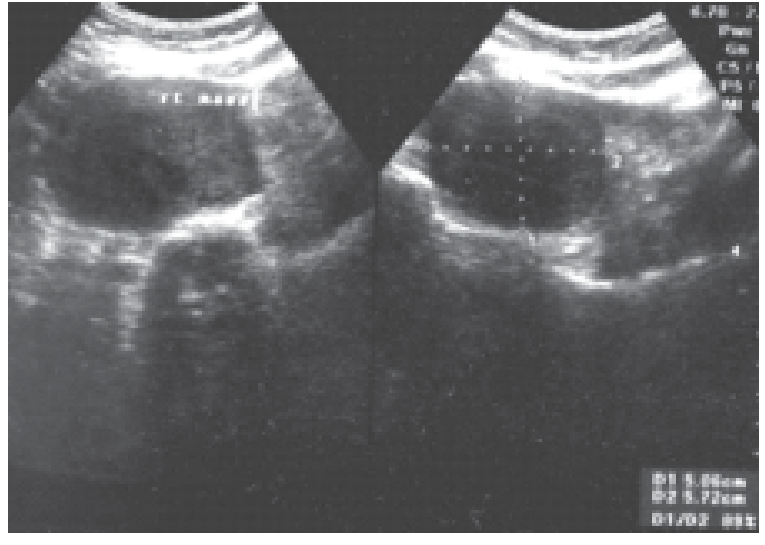

Fig.-1: USG Showed Adnexal mass

Computerized tomography showed an oval soft tissue density mass measuring about $(81.5 \times 69.6 \times 58.6) \mathrm{mm}$ having internal hypodensity representing necrosis seen in right side of pelvic cavity. Posteromedially the mass abutting the uterus and indent the urinary bladder at its superior surface. After IV contrast strong heterogeneous enhancement of the mass is noted. Radiological opinion was suggestive of pelvic mass possibly right adnexal origin.Serum CA-125, $\mathrm{LDH}$ and aFeto protein level was within range. Right sided salpingo-oopherectomy was done with no intraoperative pathologic diagnosis.

1. Registrar, Dept. of Obs. \& Gynae, Shaheed Suhrawardy Medical College \& Hospital, Dhaka.

2. Professor, Dept. of Obs. \& Gynae, Shaheed Suhrawardy Medical College \& Hospital, Dhaka.

3. Associate Professor, Dept. of Obs. \& Gynae, Shaheed Suhrawardy Medical College \& Hospital, Dhaka.

4. Registrar, Dept. of Obs. \& Gynae, Shaheed Suhrawardy Medical College \& Hospital, Dhaka.

5. Registrar, Dept. of Obs. \& Gynae, Shaheed Suhrawardy Medical College \& Hospital, Dhaka. 


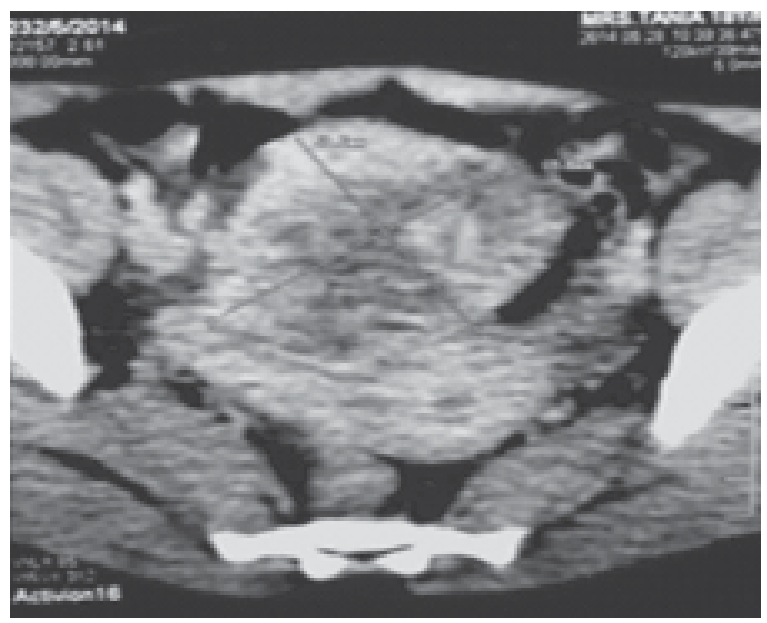

Fig.-2: Pre and post contrast CT Scan of whole abdomen showed right adnexal mass

\section{Histopathology Report}

\section{Gross examination}

Specimen consists of a gray white nodular piece of tissue measuring about $9 \times 6 \times 3 \mathrm{~cm}$. Cut surface showed yellowish and cystic area.

\section{Microscopic examination}

Section revealed round to oval cells having vaculated cytoplasm with small, dark nuclei. Spindle cells, ectatic blood vessels and fibrous components were also seen. No malignant cells were seen.

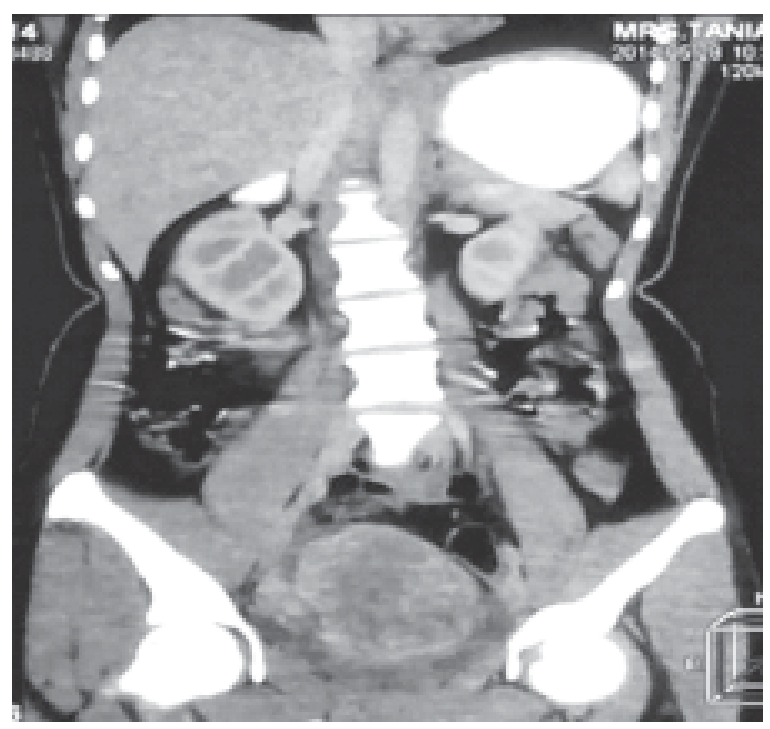

Fig.-3: Diagnosis: Sclerosing stromal tumour.

\section{Discussion:}

Sclerosing stromal tumor is a benign subtype of ovarian sex cord stromal tumor, described as a distinct entity in 1973 by Chalvardjian and Scully 4 . Sex cord stromal tumors represent approximately $8 \%$ of ovarian neoplasms ${ }^{5}$ and SST comprises less than $5 \%$ of sex cord stromal tumors. This relatively rare tumor characteristically differentiates itself histologically and clinically from others. The common presenting symptoms of SST include menstrual irregularity, pelvic pain and non-specific symptoms related to ovarian mass. Masculinisation or anovulation may be present in some patients as they are occasionally associated with oestrogen and rarely androgen secretion. SST usually presents in the 2 nd-3rd decade of life, whereas other ovarian stromal tumors present in the 5th-6th decade of life ${ }^{6}$.

Microscopic picture of SST is heterogenous and contrasts with the relative homogeneity of other stromal tumors like thecoma and fibroma. Histologically it is characterized by cellular psuedolobules, prominent interlobular fibrosis, marked vascularity and dual cell population, collagen producing spindle cells, and lipid containing round or ovoid cells ${ }^{7}$.

The vascular sclerotic and edematous stromal changes are constant features of these tumours and relate to the local elaboration of some vascular permeability and growth factors (VPF/VEGF) ${ }^{8}$. Vascular tumours are also included in the differential diagnosis due to prominent vascularity, but inhibin positivity suggests the diagnosis of sclerosing stromal tumour. Sometimes massive ovarian edema may be confused with sclerosing stromal tumours but can be differentiated by preserved ovarian tissue within edematous stroma and absence of heterogeneity. Moreover, the edema in sclerosing stromal tumor is zonal in contrast to that seen in massive edema or an edematous fibroma. Sometimes the edematous stroma of these tumors contains vacuolated cells and signet ring cells (as seen in our case), which can be mistaken for signet ring cells of Krukenbergtumour of ovary. But they can be differentiated as the latter are mostly bilateral, occur usually in the 6th and 7th decades and lack pseudo-lobular pattern of sclerosing stromal tumour. Furthermore, signet ring cells of Krukenbergtumour contain mucin rather than lipid and the cells may exhibit nuclear atypia and mitotic activity. Immunohistochemical analysis in SST shows positivity for predominant smooth muscle actin and inhibin and vimentin suggesting stromal origin of sclerosing stromal tumours ${ }^{9}$.

Calcitonin, inhibin, CD34, and á-glutathione Stransferase (áGST) positivity has been reported to be useful to differentiate sclerosing stromal tumors from thecomas, fibromas and other sex cord stromal tumors. 
Inhibin has been shown to be useful marker for ovarian sex cord stromal tumors. CD34 stains the endothelium of often dilated and branching vascular architecture and clearly distinguishes SST from thecoma and fibroma.áGST positivity within scattered cells appears to be useful in the distinction of SST from diffuse staining thecomas and no staining fibromas ${ }^{10}$.

On ultrasonography the appearances of SST may mimic that of malignant ovarian tumors because they show mixed pattern of cystic and solid components ${ }^{11}$. However color Doppler ultrasonography of SST reveals prominent vascularity in the peripheral portion and central intercystic spaces ${ }^{12}$. Magnetic resonance imaging is more helpful in differentiating SST from malignant ovarian tumors, which include a mass with hyperintense cystic components or a heterogenous solid mass of intermediate-to-high signal intensity on T2 weighted MRI. Dynamic contrast-enhanced images can even distinguish SST from other sex cord stromal tumors with striking early peripheral enhancement reflecting cellular areas with prominent vascular networks and an area of prolonged enhancement in inner portion of the mass representing collagenous hypocellular area. These findings are not a feature of thecomas and fibromas. This shows that MRI is useful in making a preoperative diagnosis of SST and distinguishing SST from other malignant ovarian tumors as well as other stromal tumors ${ }^{13}$. We stress the importance of being familiar with sclerosing stromal tumors when evaluating ovarian neoplasms in children and adolescents in order to contribute to the appropriate clinical management preventing extensive and unnecessary surgery, and preserving fertility.

\section{Conclusion:}

Due to the rarity of this particular ovarian neoplasm it is not always possible to predict the presence of this tumor preoperatively on the basis of clinical and sonographic findings. But a possibility of sclerosing stromal tumor should be kept in young patients with ovarian mass, as all of the sclerosing stromal tumors of the ovary reported in the literature were benign and were treated successfully by enucleation or unilateral ovariotomy.

\section{References:}

1. A. Chalvardjian and R. E. Scully, "Sclerosing stromal tumors of the ovary," Cancer. 1973;31(3):664-670.

2. G. Marelli, S. Carinelli, A. Mariani, L. Frigerio, and A. Ferrari, "Sclerosing stromal tumor of the ovary-report of eight cases and review of the literature," European Journal of Obstetrics
Gynecology and Reproductive Biology.1998; 76(1):85-89.

3. J. Prat, Pathology of the Ovary, Saunders, London, UK, 2004.

4. A. Chalvardjian and R. E. Scully, "Sclerosing stromal tumors of the ovary," Cancer. 1973; 31(3):664-670.

5. E. K. Outwater, B. J. Wagner, C. Mannion, J. K. McLarney, and B. Kim, "Sex cord-stromal and steroid cell tumors of the ovary," Radiographics. 1998;18(6):1523-1546.

6. S. M. Ismail and S. M. Walker, "Bilateral virilizingsclerosing stromal tumours of the ovary in a pregnant woman with Gorlin's syndrome: implications for pathogenesis of ovarian stromal neoplasms,"Histopathology.1990; 17(2): 159-163.

7. W. J. Hoskins, C. A. Perez, R. C. Young, R. R. Barakat, M. Markman, and M. E. Randall, Principles and Practice of Gynaecologic Oncology, Lippincot Williams and Wilkins, Philadelphia, Pa, USA, 4th edition, 2005.

8. S. Kawauchi, T. Tsuji, T. Kaku, T. Kamura, H. Nakano, and M. Tsuneyoshi, "Sclerosing stromal tumor of the ovary: a clinicopathologic, immunohistochemical, ultrastructural, and cytogenetic analysis with special reference to its vasculature," American Journal of Surgical Pathology.1998; 22(1):83-92.

9. A. J. Tiltman and Z. Haffajee, "Sclerosing stromal tumors, thecomas, and fibromas of the ovary: an immunohistochemical profile," International Journal of Gynecological Pathology. 1999; 18(3):254-258.

10. M. Akbulut, N. Colakoglu, M. E. Soysal, and S. E. Duzcan, "Sclerosing stromal tumor of the ovary: report of a case and review of the literature," Agean Pathology Journal. 2004;1:84-89.

11. I. Joja, K. Okuno, M. Tsunoda et al., "Sclerosing stromal tumor of the ovary: US, MR, and dynamic MR findings," Journal of Computer Assisted Tomography. 2001; 25(2):201-206.

12. B. Deval, A. Rafii, E. Darai, D. Hugol, and J. N. Buy, "Sclerosing stromal tumor of the ovary: Color Doppler findings," Ultrasound in Obstetrics and Gynecology.2003; 22(5):531-534.

13. H. Yerli, A. M. Agildere, B. Bilezikci, and E. Karadeli, "Sclerosing stromal tumor of the ovary with torsion: MRI features," ActaRadiologica. 2003; 44(6):612-615. 